\title{
Mise en œuvre d'un réseau intégré de prévention et de gestion du risque cardiométabolique en première ligne à Montréal : une plus grande coordination des soins avec les médecins de première ligne a-t-elle un impact sur les résultats de santé?
}

\author{
Sylvie Provost, M.D., M. Sc. (1,2,3); Raynald Pineault, M.D., Ph. D. (1,2,3,4); Dominique Grimard M. Sc. (1); \\ José Pérez, M. Sc. (1,2); Michel Fournier, M. Sc. (1); Yves Lévesque, M.D., M. Sc. A. (1); Johanne Desforges, M.D. (5,6); \\ Pierre Tousignant, M.D., M. Sc. (1,2,3,7); Roxane Borgès Da Silva, Ph. D. (3,8)
}

Cet article a fait l'objet d'une évaluation par les pairs.

\begin{abstract}
Résumé
Introduction : La gestion des maladies chroniques nécessite une grande intégration des services. Un programme de gestion du risque cardiométabolique inspiré du Chronic Care Model a été implanté à Montréal pour les patients atteints de diabète ou d'hypertension. Un des objectifs de notre étude était d'apprécier l'impact de la coordination des soins entre les équipes interdisciplinaires et les médecins sur la participation des patients au programme et sur l'amélioration des habitudes de vie et le contrôle de la maladie.
\end{abstract}

Méthodologie : Nous avons utilisé des données sur les résultats de santé issues d'un registre de données cliniques et de questionnaires aux patients à leur entrée dans le programme et à 12 mois de suivi, ainsi que des données sur les caractéristiques du programme provenant de l'analyse de son implantation. Nous avons réalisé des analyses de régression multiple, contrôlant pour les caractéristiques sociodémographiques et de santé des patients, pour mesurer l'association entre la coordination de l'équipe interdisciplinaire avec les médecins de première ligne et différents résultats de santé.

Résultats : Au total, 1689 patients ont participé à l'évaluation (taux de participation $60,1 \%)$. Environ $40 \%$ des patients ont abandonné le programme durant la première année. À 12 mois de suivi ( $\mathrm{n}=992)$, nous avons observé une augmentation significative de la proportion des patients atteignant les différentes cibles cliniques. La perception par l'équipe interdisciplinaire d'une meilleure coordination des soins avec les médecins de première ligne était associée à une plus grande participation des patients au programme et à l'atteinte de meilleurs résultats cliniques.

Conclusion : Une plus grande coordination des services aux patients entre des équipes interdisciplinaires et les médecins de première ligne se traduit par des effets bénéfiques chez les patients.

Mots-clés : maladies chroniques, Chronic Care Model, services de première ligne, pratique médicale, coordination des soins
Points saillants

- Le programme de gestion du risque cardiométabolique en première ligne mis en œuvre à Montréal améliore les résultats de santé chez les patients atteints de diabète ou d'hypertension.

- Une plus grande coordination des soins entre les équipes interdisciplinaires et les médecins traitants de première ligne se traduit par de meilleurs résultats chez les patients.

- Une plus grande proximité entre équipes interdisciplinaires et médecins de première ligne, ce que préconisent les nouveaux modèles d'organisation de première ligne, constitue probablement aussi une voie à privilégier pour la gestion des maladies chroniques.

\section{Introduction}

La gestion des maladies chroniques nécessite une intégration des services destinés aux personnes qui en sont atteintes ${ }^{1-3}$. Parmi les modèles de gestion des soins développés pour améliorer la prise en charge, le Chronic Care Model (CCM) est le plus utilisé. Il repose sur une intégration des services des divers niveaux du système de soins et s'articule autour de six éléments

Rattachement des auteurs :

1. Direction de santé publique du Centre intégré universitaire de santé et services sociaux (CIUSSS) du Centre-Sud-de-l'île-de-Montréal, Montréal (Québec), Canada

2. Centre de recherche du Centre hospitalier de l'Université de Montréal, Montréal (Québec), Canada

3. Institut de recherche en santé publique de l'Université de Montréal, Montréal (Québec), Canada

4. Institut national de santé publique du Québec, Montréal (Québec), Canada

5. CIUSSS du Centre-Sud-de-l'île-de-Montréal, Montréal (Québec), Canada

6. Groupe de médecine de famille et Unité de médecine familiale de Verdun, Montréal (Québec), Canada

7. Department of Epidemiology, Biostatistics and Occupational Health, McGill University, Montréal (Québec), Canada

8. Faculté des sciences infirmières de l'Université de Montréal, Montréal (Québec), Canada

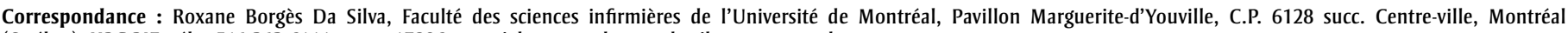
(Québec) H3C 3J7; tél. : 514-343-6111, poste 17286; courriel : roxane.borges.da.silva@umontreal.ca 
interreliés portant sur différents aspects de l'organisation des soins ${ }^{3-5}$. Un de ces éléments est l'organisation de l'offre et de la prestation de services par la mise en place d'équipes multidisciplinaires coordonnées et intégrées, dans lesquelles le partage des informations cliniques est systématique. Plusieurs études indiquent que les interventions basées sur le Chronic Care Model améliorent les processus et résultats de soins, particulièrement dans le cas du diabète ${ }^{5-9}$.

Dans le cadre de la réforme des services de santé entreprise au Québec au cours des dernières années, les Centres de santé et de services sociaux (CSSS) ont eu pour mandat d'améliorer l'accessibilité, la continuité et la coordination des services à la population, notamment en favorisant la collaboration interprofessionnelle et interorganisationnelle au sein des réseaux locaux de services, incluant les services médicaux de première ligne $\mathrm{e}^{10,11}$. Il faut noter à ce propos qu'au Québec, les services médicaux de première ligne ne sont pas complètement intégrés du point de vue organisationnel aux autres services publics de santé. L'implantation d'initiatives basées sur le CCM constitue une méthode privilégiée de consolidation des réseaux locaux de services. La participation des médecins de première ligne, acteurs-clés dans la gestion des maladies chroniques ${ }^{3,12-15}$, à des réseaux intégrés de services comporte non seulement des bénéfices pour leurs patients, mais constitue aussi un soutien précieux pour leur pratique ${ }^{16}$.

C'est dans ce contexte que l'Agence de la santé et des services sociaux de Montréal, en collaboration avec les CSSS*, a mis en œuvre en 2011 un programme de gestion du risque cardiométabolique en première ligne pour les patients atteints de diabète ou d'hypertension artérielle (HTA). Le programme est inspiré du CCM et vise la modification des habitudes de vie, le contrôle de la maladie, la prévention des complications et le soutien à l'autogestion. Dans chaque CSSS, le programme prévoit :

- un centre de formation offrant aux patients, en complémentarité avec le suivi du médecin de première ligne, des interventions cliniques orientées vers la connaissance, la motivation, l'autogestion de la maladie et la modification des habitudes de vie. Ces interventions sont réalisées par une équipe interdisciplinaire auprès de patients référés par leur médecin de famille. Pour chaque patient, le programme inclut, sur 2 ans, des rencontres de suivi individuel avec une infirmière et avec une nutritionniste, ainsi que des rencontres de groupe avec l'équipe interdisciplinaire (infirmière, nutritionniste, kinésiologue, pharmacien et travailleur social). La séquence des interventions cliniques du programme est présentée dans le tableau 1. De l'information détaillée sur le contenu des interventions est disponible sur demande auprès des auteurs;

- un soutien aux médecins de première ligne (activités de formation médicale continue, résumé des lignes directrices, information écrite à remettre aux patients et outils de référence au programme);

- l'établissement de liens avec les spécialistes et entre les partenaires du réseau local;

- la mise en place de mécanismes de coordination des soins, notamment à l'aide de l'implantation d'un registre régional informatisé des maladies chroniques pour faciliter le circulation de l'information clinique entre les intervenants.

TABLEAU 1

Séquence des interventions cliniques du programme sur le risque cardiométabolique, Montréal, Canada

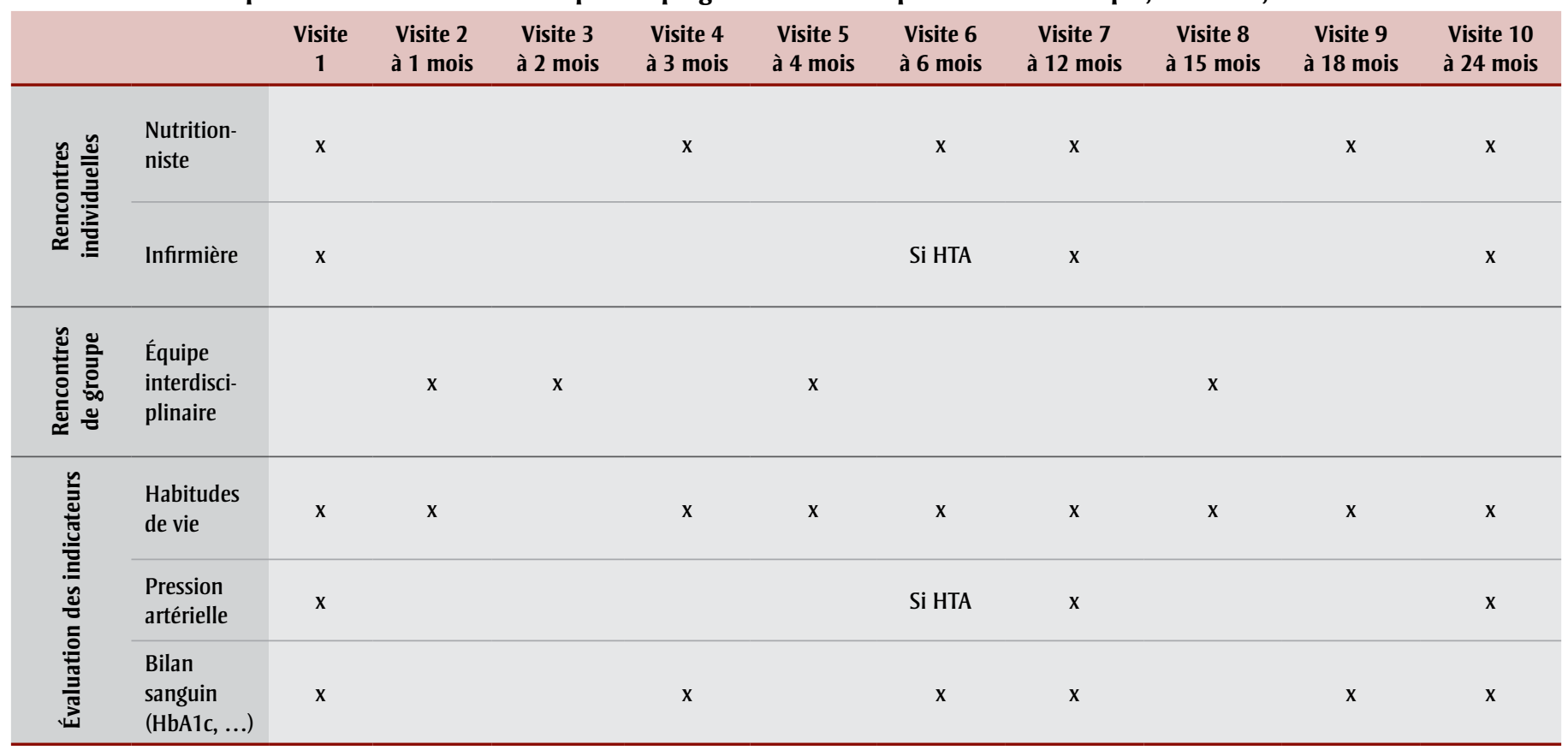

Abréviation : HTA, hypertension artérielle.

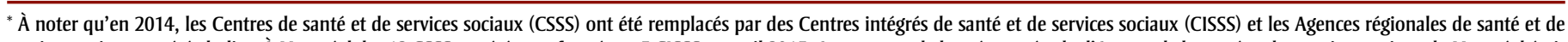

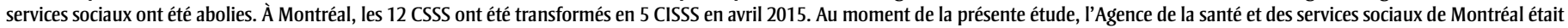
l'instance régionale responsable de la planification et de la coordination du programme à Montréal et les CSSS étaient les instances locales responsables de l'implantation du programme. 
Les objectifs de l'évaluation de l'implantation de ce programme ${ }^{17}$ étaient : apprécier le degré de mise en œuvre du programme dans les territoires de CSSS participants et identifier les facteurs contextuels pouvant l'expliquer, évaluer les effets des différents aspects de l'implantation du programme sur certains indicateurs de santé des patients, documenter la participation des médecins au programme et leur appréciation de ses effets sur leurs patients et sur leur pratique et enfin évaluer l'impact de l'implantation du programme sur la consolidation des réseaux locaux de services. Dans le cadre de cette évaluation, l'objectif de notre article est d'analyser l'impact de la coordination des soins entre les équipes interdisciplinaires des CSSS et les médecins traitants en première ligne sur la participation des patients au programme, l'amélioration de leurs habitudes de vie et le contrôle de leur maladie.

\section{Méthodologie}

\section{Devis}

L'évaluation de l'implantation du programme sur le risque cardiométabolique est basée sur un devis mixte. Nous avons réalisé l'analyse d'implantation du programme à l'aide d'une stratégie de recherche qualitative (entrevues semi-dirigées, collecte de documents officiels, questionnaires aux gestionnaires et intervenants impliqués dans chaque CSSS). Les résultats détaillés de cette analyse d'implantation seront présentés dans un autre article. Pour analyser les effets du programme chez les patients, nous avons utilisé une approche quantitative, apparentée à un devis quasiexpérimental " avant-après ». Nous avons utilisé les données provenant de questionnaires auto-administrés aux patients, complétés sur place à leur entrée dans le programme (T0) et à 12 mois de suivi (T12), ainsi que du registre informatisé des maladies chroniques dans lequel les intervenants ont entré les données cliniques à chaque rencontre avec les patients. Six des 12 CSSS de Montréal ont participé à l'évaluation du programme. La sélection des CSSS participants reposait sur le volontariat et sur leur engagement à respecter le cadre général d'implantation du programme proposé par l'Agence. La collecte des données s'est déroulée de mars 2011 à août 2014.

\section{Définition des variables et sources de données}

\section{Variables dépendantes}

Les résultats (" outcomes ») étudiés ou variables dépendantes portaient sur la participation au programme et sur certains résultats de santé des patients. Trois variables décrivaient la participation des patients au programme : les abandons, le degré d'exposition au programme et le degré de respect du calendrier. Les abandons considérés dans l'analyse étaient les " abandons confirmés ", c'est-à-dire ceux explicitement signifiés par les patients aux intervenants des CSSS. Le degré d'exposition au programme, documenté à l'aide des données du registre sur les maladies chroniques, correspondait au nombre d'interventions (rencontres avec la nutritionniste, rencontres avec l'infirmière, cours de groupe) réalisées depuis l'entrée du patient dans le programme, sans tenir compte de leur conformité à la séquence des interventions prescrite dans le calendrier du programme. Le degré de respect du calendrier, pour chaque patient, était quant à lui représenté par le pourcentage des interventions (nutrition, infirmière, cours de groupe) ayant effectivement eu lieu dans les temps de suivi prévus au calendrier depuis l'entrée du patient dans le programme. Les temps de suivi ont été définis pour chaque patient en fonction de sa date d'entrée dans le programme en y ajoutant un intervalle pour prendre en compte le fait que les visites n'avaient pas nécessairement toujours lieu au moment précis prescrit dans le calendrier.

Pour chaque patient, les résultats de santé étudiés portaient sur le contrôle de la maladie (atteinte des cibles d'hémoglobine glyquée [HbA1c] et de tension artérielle [TA]) et sur les habitudes de vie (niveau d'activité physique et répartition des glucides alimentaires), ainsi que sur l'impact de la maladie sur la qualité de vie. Nous avons extrait les données sur le contrôle de la maladie et sur les habitudes de vie du registre sur les maladies chroniques. Les valeurs d'HbA1c ont été mesurées à partir de prélèvements sanguins effectués en laboratoire et inscrites dans le registre par l'infirmière ou la nutritionniste. La variable d'atteinte de la cible d'HbA1c a été dichotomisée : $7 \%$ ou moins et plus de $7 \%$. Les valeurs de TA ont été mesurées par l'infirmière du programme à l'aide d'un sphygmomanomètre automatisé et inscrites par celle-ci dans le registre. La variable d'atteinte de la cible de TA a été dichotomisée : moins de 140/90 (ou moins de 130/80 chez les patients atteints de diabète) et 140/90 ou plus (ou 130/80 ou plus chez les patients atteints de diabète). Les données concernant l'atteinte de la cible d'activité physique provenaient d'un bref questionnaire adapté de celui de l'Enquête québécoise sur l'activité physique et la santé18 complété à chaque visite du patient; ces données étaient inscrites dans le registre par la nutritionniste ou la kinésiologue. Cet indicateur mesurait sur une échelle de 1 à 4 le nombre de jours par semaine où le patient avait fait au moins 30 minutes d'activité physique, pondéré par l'intensité de ces activités physiques. La variable d'atteinte de la cible d'activité physique a été dichotomisée ( 3 ou 4 sur l'échelle de 4 , et moins de 3 sur l'échelle de 4). La mesure de la variable de répartition des glucides était basée sur l'évaluation de l'alimentation du patient effectuée par la nutritionniste à chaque visite, en fonction des objectifs de répartition quotidienne des glucides alimentaires présentés dans le document Coup d'œil sur l'alimentation de la personne diabétique ${ }^{19}$, et exprimée en deux catégories : répartition équilibrée, oui ou non.

Les questions relatives à la mesure de l'impact de la maladie sur la qualité de vie des patients ont été adaptées du questionnaire Audit of Diabetes Dependent Quality of Life ${ }^{20-22}$. Elles mesuraient la perception des patients de ce à quoi ressembleraient les différentes dimensions de leur vie s'ils n'étaient pas atteints de diabète ou d'hypertension, sur une échelle allant de " considérablement meilleure " à " semblable ". Les dimensions mesurées étaient les opportunités d'emploi ou de carrière, la vie sociale, la vie sexuelle, les possibilités d'activités sportives, de vacances, de voyage ou de loisirs, la motivation à accomplir des choses, la capacité à accomplir des activités physiques et le plaisir de manger. Pour chaque patient, un indice d'impact de la maladie sur la qualité de vie a été calculé en faisant la moyenne des réponses aux différents éléments, pondérée par l'importance (de « très important » à " pas du tout important ») aux yeux du patient de chacune de ces dimensions de la vie, et rapporté sur 10 .

\section{Variables explicatives}

Les deux variables explicatives de l'étude, mesurées à partir des informations recueillies auprès des gestionnaires et intervenants des CSSS lors de l'analyse d'implantation, 
concernaient la « coordination externe » du programme. Ces variables rendaient compte de la coordination des soins et du suivi interdisciplinaire entre l'équipe du CSSS et le médecin de famille assurant le suivi médical du patient en première ligne pour son diabète ou son HTA. La première variable, documentée par questionnaire (et confirmée lors des entrevues semi-dirigées) auprès des gestionnaires du programme dans les CSSS, portait sur la fréquence $d u$ retour d'information clinique aux médecins. Les CSSS ont été classés en trois catégories : fréquence élevée (communication écrite au médecin d'emblée après chaque rencontre individuelle ou cours de groupe, ou plus souvent si nécessaire), moyenne (communication d'emblée aux 6 mois, ou plus souvent si nécessaire) et faible (une fois par année ou moins, ou au besoin seulement).

La seconde variable portait sur la perception des intervenants des CSSS au sujet de la réalisation du suivi interdisciplinaire et de la coordination des soins avec les médecins. Dans le cadre de l'analyse d'implantation, un bref questionnaire a été administré aux intervenants de l'équipe dans chaque CSSS (leur nombre variant entre 5 et 10), dans lequel on leur a demandé d'évaluer, sur une échelle de 1 à 5 ( 5 correspondant à " très élevé » et 1 à " très faible »), le degré de réalisation avec les médecins traitants des patients des éléments suivants : coordination des soins, complémentarité des soins, suivi interdisciplinaire sur le cheminement et les progrès du patient, suivi interdisciplinaire sur les résultats de laboratoire et suivi interdisciplinaire au besoin lorsque le patient devient instable. Les CSSS ont été classés en deux catégories en fonction de la perception des intervenants quant au suivi interdisciplinaire et à la coordination des soins avec les médecins : élevée $(60 \%$ ou plus des intervenants ont répondu 4 ou 5 à au moins deux des cinq questions) et faible (moins de $60 \%$ ont répondu 4 ou 5 à au moins deux des cinq questions).

\section{Variables de contrôle}

Parmi les variables de contrôle utilisées dans les analyses, celles sur les caractéristiques individuelles des patients provenaient du questionnaire administré à leur entrée dans le programme : âge, sexe, origine, langue parlée à la maison, niveau de scolarité, occupation, perception de l'état de santé et nombre de comorbidités (problème cardiaque, accident cérébrovasculaire, asthme ou maladie pulmonaire obstructive chronique, arthrite/arthrose ou problèmes de santé mentale). Le type de clinique de première ligne décrivait l'organisation de services médicaux de première ligne où le patient était suivi pour son diabète ou son HTA et a été défini à partir des données d'inscription des patients au programme. Les types de clinique étaient : groupe de médecine de famille (GMF), cliniqueréseau (CR), GMF-CR, Centre local de services communautaires (CLSC), unité d'enseignement en médecine familiale, clinique de groupe non GMF ni CR, ou clinique solo. La mesure de l'indice de masse corporelle (IMC) à T0 provenait du registre de maladies chroniques. Dans les modèles d'analyse des résultats en lien avec la participation des patients au programme (abandon, exposition au programme et respect du calendrier), le score d'impact de la maladie sur la qualité de vie à T0 a aussi été inclus dans les covariables, de même que le score de connaissance de la maladie à T0. Ce dernier a été calculé en faisant la moyenne des réponses à quatre questions adaptées des travaux de Battersby et collab. ${ }^{23}$ mesurant, sur une échelle de 1 à 4 (allant de "pas du tout en accord " à « beaucoup en accord »), le fait d'avoir une bonne connaissance de sa maladie (diabète ou HTA), une bonne connaissance de son traitement, d'être capable de déceler les signes et symptômes indiquant un changement dans l'évolution de sa maladie et de savoir quoi faire en cas d'apparition de ces signes ou symptômes.

\section{Méthode d'analyse}

Nous avons construit des modèles d'analyse de régression multiple, contrôlant pour les caractéristiques sociodémographiques et de santé des patients à T0, pour mesurer l'association entre chacune des variables dépendantes étudiées et 1) la fréquence du retour d'information clinique aux médecins de première ligne et 2) la perception des intervenants des CSSS au sujet du suivi interdisciplinaire et de la coordination des soins avec les médecins de première ligne. Selon la nature des variables dépendantes, nous avons utilisé des modèles logistiques, linéaires ou des régressions de Poisson.

Les données ont été analysées à l'aide du logiciel Stata version 13 (StataCorp LP, College Station, Texas, États-Unis). Le projet a reçu l'approbation du comité d'éthique de la recherche de l'Agence de la santé et des services sociaux de Montréal.

\section{Résultats}

$\mathrm{Au}$ total, parmi les 2810 patients inscrits au programme entre mars 2011 et août 2013, 1689 ont participé à l'évaluation (taux de participation de 60,1 \%). Parmi eux, environ $40 \%$ ont abandonné le programme au cours de la première année (à $\mathrm{T} 12, \mathrm{n}=992)$. Une proportion importante de patients ayant abandonné (41 \%) l'ont fait après leur première visite et près du tiers (31 \%) des patients ayant abandonné ont déclaré que le programme demandait trop de temps ou que l'horaire ne convenait pas. Les caractéristiques sociodémographiques et de santé des patients ayant un suivi de 12 mois ou plus dans le programme sont présentées dans le tableau 2. Parmi ces patients, plus de la moitié $(56,9 \%)$ étaient des femmes et l'âge moyen était de 58,5 ans. À T0, plus de $60 \%$ avaient une HbA1c de $7 \%$ ou moins, plus de $40 \%$ avaient une TA inférieure à 140/90 (<130/80 chez les patients atteints de diabète), mais près des deux tiers avaient un IMC de 30 ou plus et souffraient d'au moins une comorbidité.

À 12 mois de suivi, le degré moyen d'exposition des patients aux interventions du programme était d'environ $75 \%$ du nombre d'interventions prévues par le programme, le degré moyen de respect du calendrier se situant pour sa part à environ $60 \%$. Les patients plus âgés, ainsi que les patients ayant un niveau de scolarité plus élevé et les patients sans comorbidité, avaient un degré de respect du calendrier plus élevé et leur taux d'abandon avant 12 mois était plus faible (données non présentées).

Les résultats présentés dans le tableau 3 indiquent qu'à 12 mois de suivi, on observait une augmentation significative de la proportion de patients ayant atteint les cibles de répartition équilibrée des glucides, d'HbA1c de $7 \%$ ou moins et de TA inférieure à 140/90 (< 130/80 chez les patients atteints de diabète), ainsi qu'une diminution significative de l'impact de la maladie sur la qualité de vie (score moyen passant de 4,56 à 4,04 sur 10). Toutefois, malgré une augmentation significative à 3 et à 6 mois de suivi (données non illustrées), la proportion à 12 mois de patients ayant atteint la cible d'activité physique n’avait pas augmenté de façon significative. 
TABLEAU 2

Caractéristiques, à leur entrée dans le programme, des patients ayant un suivi à 12 mois $(\mathbf{n}=992)$, Montréal, Canada, 2011-2014

\begin{tabular}{|c|c|c|}
\hline Caractéristiques sociodémographiques des patients à T0 & & $\%^{\mathrm{a}}$ \\
\hline Sexe & Femmes & 56,9 \\
\hline Groupe d'âge & Moins de 55 ans & 34,0 \\
\hline & 65 ans ou plus & 31,7 \\
\hline Origine & Nés au Canada & 72,5 \\
\hline Niveau de scolarité & Moins d'un secondaire 5 & 12,9 \\
\hline (plus haut niveau de diplôme obtenu) & Diplôme de secondaire 5 & 45,8 \\
\hline & Diplôme d'études collégiales & 13,7 \\
\hline & Diplôme universitaire & 27,6 \\
\hline & HTA sans diabète/prédiabète & 4,4 \\
\hline & Diabète/prédiabète avec HTA & 87,6 \\
\hline HbA1c (pour les patients avec diabète ou prédiabète) & $\leq 7 \%$ & 62,7 \\
\hline & $7 \%$ à $8 \%$ & 18,5 \\
\hline & $>8 \%$ & 18,8 \\
\hline TA (tous les patients) & $<140 / 90$ (ou < 130/80 si diabète) & 41,0 \\
\hline IMC & $<25$ & 8,1 \\
\hline & 25 à $<30$ & 28,1 \\
\hline Comorbidités & Problème cardiovasculaire & 17,3 \\
\hline & Asthme/MPOC & 21,0 \\
\hline & Arthrite/arthrose & 37,8 \\
\hline & Problème de santé mentale & 22,3 \\
\hline Nombre de comorbidités & Aucune & 34,0 \\
\hline & 1 & 37,4 \\
\hline & 2 ou plus & 28,6 \\
\hline Type de suivi en première ligne & & \\
\hline Type de clinique où le patient est suivi pour son pré/diabète & GMF, CR ou GMF-CR & 58,6 \\
\hline ou son HTA & CLSC ou UMF (non GMF ou CR) & 15,3 \\
\hline & $\begin{array}{l}\text { Groupe ou pratique solo (incluant un faible pourcentage de patients sans } \\
\text { médecin de famille à T0) }\end{array}$ & 26,1 \\
\hline
\end{tabular}

Abréviations : CLSC, centre local de services communautaires; CR, clinique réseau; GMF, groupe de médecine de famille; HbA1c, hémoglobine glyquée; HDL-C, «high-density lipoprotein cholesterol »; HTA, hypertension artérielle; IMC, indice de masse corporelle; MPOC, maladie pulmonaire obstructive chronique; T0, entrée du patient dans le programme; TA, tension artérielle; TC, «total cholesterol»; UMF, unité d'enseignement en médecine familiale.

${ }^{a}$ Les données manquantes sont exclues des calculs de fréquence. 
TABLEAU 3

Évolution des habitudes de vie, de l'HbA1c, de la TA et de la perception de l'impact de la maladie sur la qualité de vie entre T0 et T12 chez les patients participant au programme à 12 mois, Montréal, 2011-2014

\begin{tabular}{|c|c|c|c|c|}
\hline & \multicolumn{2}{|c|}{$\begin{array}{l}\text { Proportion de patients ayant atteint la valeur cible } \\
\text { (en \%) }\end{array}$} & \multirow{2}{*}{$\begin{array}{l}\text { Effectifs avec mesure à } \\
\text { T0 et à T12 }\end{array}$} & \multirow[t]{2}{*}{ Valeur $p^{2}$} \\
\hline & à T0 & à T12 & & \\
\hline Répartition équilibrée des glucides & 22,2 & 35,9 & 708 & $<0,001$ \\
\hline HbA1c $\leq 7 \%$ (chez les pré/diabétiques) & 66,9 & 72,9 & 602 & $<0,001$ \\
\hline \multirow{2}{*}{ TA < 140/90 (diabétiques < 130/80) } & \multicolumn{2}{|c|}{ Score moyen sur 10} & \multirow{2}{*}{$\begin{array}{l}\text { Effectifs avec mesure à } \\
\text { T0 et à T12 }\end{array}$} & \multirow{2}{*}{ Valeur $p$} \\
\hline & à T0 & à T12 & & \\
\hline Impact de la maladie sur la qualité de vie & 4,56 & 4,04 & 481 & $<0,001$ \\
\hline
\end{tabular}

Abréviations : HbA1c, hémoglobine glyquée; T0, entrée du patient dans le programme; T12, suivi après 12 mois; TA, tension artérielle.

a Tests de comparaison de proportions et de scores entre T0 et T12 chez les patients ayant des mesures aux deux temps.

En matière de participation des patients au programme, les résultats des analyses multivariées ont indiqué que la fréquence des communications envoyées aux médecins traitants de première ligne était associée à une plus grande exposition au programme et à un plus grand respect du calendrier à 12 mois de suivi (tableau 4). Une meilleure perception de l'équipe du programme dans les CSSS au sujet du suivi interdisciplinaire et de la coordination avec les médecins de première ligne était associée à une proportion moindre d'abandons au programme et, parmi les patients ayant abandonné, à une proportion moindre d'abandons avant 3 mois de suivi (tableau 4). Les résultats de santé, à l'exception du niveau d'activité physique, étaient quant à eux associés positivement à une meilleure perception de l'équipe du programme au sujet du suivi interdisciplinaire et de la coordination avec les médecins de première ligne (tableau 5).

\section{Analyse}

À l'instar d'autres études qui suggèrent que l'utilisation d'interventions basées sur le CCM permet d'améliorer le contrôle glycémique et la tension artérielle des patients atteints de diabète ${ }^{5-9}$, nos résultats indiquent que la mise en œuvre du programme sur le risque cardiométabolique à Montréal s'est traduite par des résultats de santé positifs pour les patients, notamment en matière

TABLEAU 4

Association entre la coordination externe avec les médecins de première ligne et la participation des patients au programme sur le risque cardiométabolique, Montréal, 2011-2014

\begin{tabular}{|c|c|c|c|c|c|c|c|c|c|}
\hline \multirow{4}{*}{ Variables explicatives } & & \multicolumn{8}{|c|}{ Résultats étudiés («outcomes»)a } \\
\hline & & \multirow{2}{*}{\multicolumn{2}{|c|}{$\begin{array}{l}\text { Abandons confirmés avant } \\
\qquad \begin{array}{c}\text { T12 } \\
\text { n }=1015^{\mathrm{c}}\end{array}\end{array}$}} & \multirow{2}{*}{\multicolumn{2}{|c|}{$\begin{array}{l}\text { Abandons confirmés } \\
\text { précoces (avant T3) }^{\mathrm{d}} \\
\qquad \mathrm{n}=388^{\mathrm{c}}\end{array}$}} & \multirow{2}{*}{\multicolumn{2}{|c|}{$\begin{array}{c}\text { Exposition } \\
\text { au programme (nombre } \\
\text { d'interventions/10) à T12 } \\
\mathrm{n}=741^{\mathrm{c}}\end{array}$}} & \multirow{2}{*}{\multicolumn{2}{|c|}{$\begin{array}{l}\text { Degré }(\%) \text { de respect du } \\
\text { calendrier à T12 } \\
\qquad \mathrm{n}=741^{\mathrm{c}}\end{array}$}} \\
\hline & & & & & & & & & \\
\hline & & $\mathrm{RC}^{\mathrm{e}}$ & IC à $95 \%$ & $\mathrm{RC}^{\mathrm{e}}$ & IC à $95 \%$ & $\mathrm{RTI}^{\mathrm{e}}$ & IC à $95 \%$ & $\beta^{\mathrm{e}}$ & IC à $95 \%$ \\
\hline \multirow{2}{*}{$\begin{array}{l}\text { Fréquence des communica- } \\
\text { tions écrites envoyées aux } \\
\text { médecins } \\
\text { (réf : Faible) }\end{array}$} & Moyenne & 1,33 & 0,86 à 2,05 & 0,86 & 0,43 à 1,71 & $1,20^{*}$ & 1,11 à 1,30 & $9,21^{*}$ & 5,90 à 12,52 \\
\hline & Élevée & 1,28 & 0,84 à 1,95 & 0,85 & 0,43 à 1,67 & $1,07^{* *}$ & 0,99 à 1,15 & 0,95 & $-2,17$ à 4,06 \\
\hline $\begin{array}{l}\text { Suivi interdisciplinaire et } \\
\text { coordination des soins } \\
\text { (réf : Plus faible) }\end{array}$ & $\begin{array}{l}\text { Plus } \\
\text { important }\end{array}$ & $0,35^{*}$ & 0,22 à 0,53 & $0,30^{*}$ & 0,12 à 0,77 & 1,01 & 0,95 à 1,08 & $-0,69$ & $-3,36$ à 1,99 \\
\hline
\end{tabular}

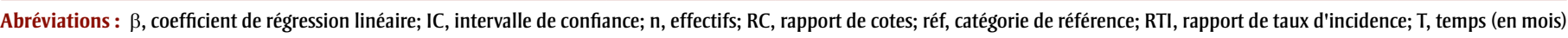
depuis l'entrée du patient dans le programme.

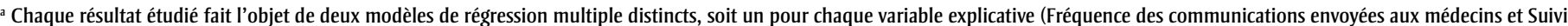
interdisciplinaire et coordination des soins).

${ }^{\mathrm{b}}$ Abandons confirmés avant T12 $=1(\mathrm{n}=513)$ et patients ayant un suivi à T12 $=0(\mathrm{n}=992)$.

c Effectifs (n) retenus effectivement dans les analyses, excluant les données manquantes.

${ }^{\mathrm{d}}$ Abandons confirmés précoces (avant T3) $=1(\mathrm{n}=290)$ et abandons confirmés à T3 ou après $=0(\mathrm{n}=378)$.

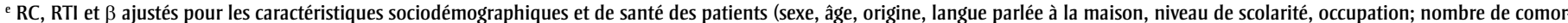

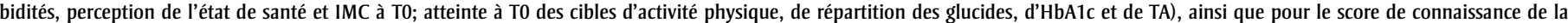
maladie à $\mathrm{T} 0$, le score d'impact de la maladie sur la qualité de vie à T0 et le type de clinique de première ligne où le patient est suivi pour son prédiabète/diabète ou son HTA.

${ }^{*} \mathrm{p}<0,05$.

${ }^{* *} \mathrm{p}<0,10$. 


\section{TABLEAU 5}

Association entre la coordination externe avec les médecins de première ligne et l'atteinte des résultats de santé à 12 mois de suivi dans le programme sur le risque cardiométabolique, Montréal, 2011-2014

\begin{tabular}{|c|c|c|c|c|c|c|c|c|c|c|c|}
\hline \multirow{4}{*}{ Variables explicatives } & & \multicolumn{10}{|c|}{ Résultats étudiés (« outcomes») à T12a } \\
\hline & & \multirow{2}{*}{\multicolumn{2}{|c|}{$\begin{array}{l}\text { Niveau d'activité } \\
\text { physique }=3 \text { ou } 4 \text { sur } \\
\text { l'échelle de } 4^{\mathrm{b}} \\
\mathrm{n}=650^{\mathrm{c}}\end{array}$}} & \multirow{2}{*}{\multicolumn{2}{|c|}{$\begin{array}{l}\text { Répartition équilibrée } \\
\qquad \begin{array}{c}\text { glucides }^{\mathrm{d}} \\
\mathrm{n}=651^{\mathrm{c}}\end{array}\end{array}$}} & \multirow{2}{*}{\multicolumn{2}{|c|}{$\begin{array}{c}\mathrm{HbA} 1 \mathrm{c} \leq 7 \% \mathrm{e}^{\mathrm{e}} \\
\mathrm{n}=554^{\mathrm{c}}\end{array}$}} & \multirow{2}{*}{\multicolumn{2}{|c|}{$\begin{array}{c}\text { TA }<140 / 90 \\
(<130 / 80 \text { si diabète })^{\mathrm{f}} \\
\mathrm{n}=564^{\mathrm{c}}\end{array}$}} & \multirow{2}{*}{\multicolumn{2}{|c|}{$\begin{array}{c}\text { Score (/10) d'impact de la } \\
\text { maladie sur la qualité de } \\
\text { vie } \\
\text { n }=404^{c}\end{array}$}} \\
\hline & & & & & & & & & & & \\
\hline & & $\mathrm{RC}^{\mathrm{g}}$ & IC à 95 \% & $\mathrm{RC}^{8}$ & IC à 95 \% & $\mathrm{RC}^{\mathrm{g}}$ & IC à 95 \% & $\mathrm{RC}^{8}$ & IC à 95 \% & $\beta^{\mathrm{g}}$ & IC à 95 \% \\
\hline \multirow{2}{*}{$\begin{array}{l}\text { Fréquence des communica- } \\
\text { tions écrites envoyées aux } \\
\text { médecins } \\
\text { (réf : Faible) }\end{array}$} & Moyenne & 0,81 & 0,48 à 1,35 & $0,23^{*}$ & 0,14 à 0,39 & 0,54 & 0,26 à 1,09 & $1,58^{* *}$ & 0,93 à 2,71 & $-0,03$ & $-0,55$ à 0,50 \\
\hline & Élevée & 0,72 & 0,43 à 1,20 & $0,30^{*}$ & 0,18 à 0,49 & 1,24 & 0,59 à 2,63 & $1,92^{*}$ & 1,13 à 3,27 & $-0,64^{*}$ & $-1,16$ à $-0,13$ \\
\hline $\begin{array}{l}\text { Suivi interdisciplinaire et } \\
\text { coordination des soins } \\
\text { (réf : Plus faible) }\end{array}$ & $\begin{array}{l}\text { Plus } \\
\text { important }\end{array}$ & 1,07 & 0,70 à 1,63 & $1,51^{*}$ & 1,01 à 2,24 & $2,51^{*}$ & 1,31 à 4,82 & $1,49^{* *}$ & 0,97 à 2,29 & $-0,55^{*}$ & $-1,01$ à $-0,09$ \\
\hline
\end{tabular}

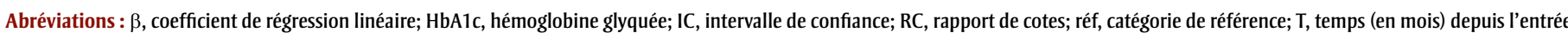
du patient dans le programme; TA, tension artérielle.

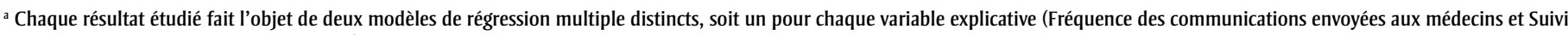
interdisciplinaire et coordination des soins).

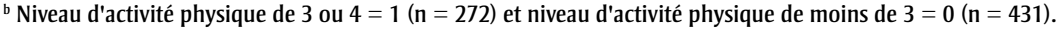

c Effectifs (n) retenus effectivement dans les analyses, excluant les données manquantes.

${ }^{\mathrm{d}}$ Répartition équilibrée des glucides $=1(\mathrm{n}=254)$ et répartition non équilibrée des glucides $=0(n=454)$.

${ }^{\mathrm{e}} \mathrm{HbA} 1 \mathrm{c} \leq 7 \%=1(\mathrm{n}=439)$ et $\mathrm{HbA} 1 \mathrm{c}>7 \%=0(n=163)$.

${ }^{\mathrm{f}} \mathrm{TA}<140 / 90(<130 / 80$ si diabète $)=1(n=288)$ et $\mathrm{TA} \geq 140 / 90(\geq 130 / 80$ si diabète $)=0(n=324)$.

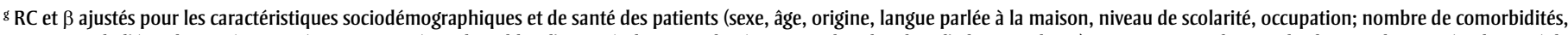

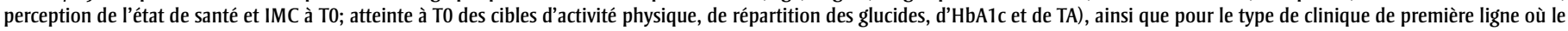
patient est suivi pour son prédiabète/diabète ou son HTA.

${ }^{*} \mathrm{p}<0,05$.

${ }^{* *} \mathrm{p}<0,10$.

de modification des habitudes de vie et d'atteinte de cibles de suivi pour l'HbA1c et la TA.

Ce programme sur le risque cardiométabolique s'inspire du CCM entre autres en mettant l'accent sur une organisation de l'offre et de la prestation de services basée sur la mise en place d'équipes multidisciplinaires coordonnées et intégrées, ainsi que sur le partage des informations cliniques. D’après nos résultats, une plus grande coordination des soins entre l'équipe interdisciplinaire du programme sur le risque cardiométabolique et les médecins traitants de première ligne, mesurée par la perception des intervenants des CSSS au sujet de la réalisation du suivi interdisciplinaire et de la coordination des soins avec les médecins, est associée à de meilleurs résultats de santé pour les patients. Il est intéressant de constater que cette association positive est présente non seulement en ce qui a trait aux résultats concernant la modification des habitudes de vie et le contrôle de l'HbAlc et de la $\mathrm{TA}$, mais aussi en matière de perception des patients relativement à l'impact de leur maladie sur leur qualité de vie. De plus, nos résultats indiquent qu'une plus grande coordination des soins avec les médecins traitants en première ligne se traduit par une meilleure participation au programme des patients inscrits, et notamment par de plus faibles taux d'abandon (et d'abandon avant 3 mois de suivi).

Dans une revue systématique de la littérature s'intéressant aux effets associés à chacune des composantes du CCM sur la prise en charge du diabète dans les soins de première ligne aux États-Unis, Stellefson et collab. ont démontré qu'aucune des composantes du CCM n'était responsable à elle seule des résultats du CCM et qu'il restait à déterminer quelle combinaison de ces composantes produit les meilleurs résultats $^{24}$. Toutefois, le fait de mettre en œuvre plusieurs composantes du modèle s'est avéré associé à de meilleurs résultats chez les patients ${ }^{5,24,25}$. Si notre étude ne permet pas de distinguer l'effet des différentes composantes du programme mis en place sur les résultats chez les patients, elle suggère que la collaboration et la coordination des soins entre des équipes interdisciplinaires et les médecins de première ligne sont bénéfiques pour les patients. Bien que nos données ne nous permettent pas de préciser le mécanisme responsable de cet effet, nous pouvons faire l'hypothèse qu'une plus grande coordination des soins se traduit par une meilleure circulation de l'information clinique concernant les patients entre les équipes interdisciplinaires et les médecins de première ligne.

Il est intéressant de noter qu'une perception favorable des intervenants des CSSS au sujet du suivi interdisciplinaire et de la coordination des soins avec les médecins semble être plus systématiquement associée à de meilleurs résultats chez les patients que la fréquence du retour d'information clinique aux médecins. Ceci suggère que la simple mise en place de mécanismes formels de communication pourrait ne pas être suffisante en soi pour favoriser l'amélioration des résultats de santé dans un tel programme, et que l'aspect qualitatif de l'interaction développée entre les équipes interdisciplinaires et 
les médecins de première ligne revêt une importance non négligeable. À cet égard, nos résultats ne montrent pas d'association significative entre une fréquence élevée du retour d'information clinique aux médecins et l'atteinte de la cible d'hémoglobine glyquée de $7 \%$ ou moins; ils font aussi état d'une association négative avec l'atteinte d'une distribution équilibrée des glucides alimentaires. Ce dernier résultat pourrait être dû à une politique de communications écrites plus régulières aux médecins dans certains CSSS ayant un nombre plus élevé de patients présentant plus de difficultés à atteindre une distribution équilibrée des glucides alimentaires.

Enfin, nos résultats indiquent l'existence d'une corrélation entre le fait que les intervenants du programme aient perçu comme plus importants le suivi interdisciplinaire et la coordination des soins avec les médecins et un taux d'abandon du programme plus faible chez les patients. Ceci pourrait s'expliquer par une transmission d'informations aux patients sur l'importance de bien suivre le programme mieux coordonnée et «cohésive» entre les intervenants et les médecins traitants dans les milieux où existe une plus grande coordination des soins.

\section{Forces et limites}

Une des forces de cette étude est, outre d'avoir été réalisée en coproduction avec les décideurs et les intervenants, d'avoir été effectuée dans un contexte clinique correspondant à la réalité vécue par les patients et par les intervenants. Les résultats obtenus sont ainsi fidèles à la réalité clinique, les rendant plus facilement généralisables à des contextes présentant des similitudes. La mise en relation de certaines caractéristiques organisationnelles du programme issues de l'analyse de l'implantation avec les résultats chez les patients constitue l'une des grandes forces de cette étude. Les différentes sources de données disponibles pour les résultats chez les patients (questionnaires, données cliniques) ainsi que les différents résultats de santé étudiés ont de plus permis de documenter les effets de la coordination des soins sur plusieurs aspects en lien avec le suivi des patients dans le programme.

Dans cette étude, la présence de certains biais ne peut être totalement exclue. Ainsi, les données sur les habitudes de vie et sur la perception de l'impact de la maladie sur la qualité de vie pourraient comporter un biais de désirabilité sociale. Par ailleurs, l'amélioration clinique générale mesurée chez les patients participants pourrait être due, au moins en partie, au fait que les patients les moins motivés aient abandonné le programme avant d'atteindre un suivi de 12 mois. Toutefois, il semble peu probable que les biais potentiels et les abandons aient influencé de façon différente les résultats du groupe de patients exposés au programme dans les CSSS où la coordination externe était plus importante et ceux du groupe exposé à une moins grande coordination externe.

Par ailleurs, les variables sur les habitudes de vie et le contrôle de l'HbA1c et de la TA comportaient une certaine proportion de données manquantes à T0 (entre 4 et $8 \%$ ). Ces données ont fait l'objet d'une imputation à l'aide de la méthode " hot deck ", consistant à remplacer chacune des valeurs manquantes par une valeur choisie aléatoirement parmi celles observées chez des patients similaires ${ }^{26}$ quant à l'âge, au sexe et à l'atteinte ou non de la cible pour la variable à imputer au prochain suivi où cette donnée était disponible. Cette approche nous a permis de réduire l'impact d'éventuels biais liés à la non-réponse ${ }^{27}$.

En ce qui concerne la mesure de la « coordination externe ", il faut rappeler que les caractéristiques organisationnelles du programme dans les CSSS qui ont été utilisées dans cette étude ont été extraites des informations recueillies lors de l'analyse d'implantation. Leur valeur est la même pour tous les patients inscrits au programme dans un CSSS donné. De plus, le nombre relativement faible de milieux cliniques participants a réduit notre capacité à observer des variations et pourrait avoir conduit à une sous-estimation de la force des associations mesurées. Par ailleurs, la mesure du suivi interdisciplinaire et de la coordination des soins entre les équipes des CSSS et les médecins repose sur la perception des intervenants des équipes. L'absence d'information sur la perception des médecins de première ligne quant à la coordination des soins avec les équipes interdisciplinaires dans les CSSS constitue une lacune.

Enfin, bien que le taux de participation à l'évaluation du programme ait été élevé, nous ne pouvons avoir la certitude que les participants soient totalement représentatifs de l'ensemble des patients inscrits au programme. Néanmoins, d'après nos données, les patients ayant participé à l'évaluation ne différaient pas de manière statistiquement significative de l'ensemble des participants au programme en matière d'âge et de sexe.

\section{Conclusion}

L'implantation du programme de prévention et de gestion du risque cardiométabolique en première ligne mis en place à Montréal constitue un exemple d'intégration des services entre des équipes interdisciplinaires et les médecins de première ligne. Même dans un contexte où les services médicaux de première ligne ne sont pas complètement intégrés aux autres services publics de santé, ce programme possède le potentiel d'une intégration réussie des services de prévention et de gestion des maladies chroniques.

Nos résultats indiquent qu'une plus grande intégration et une plus grande coordination des services entre des équipes interdisciplinaires et les médecins de première ligne, ce que préconise le CCM, se traduisent par des effets bénéfiques chez les patients atteints de diabète ou d'hypertension. Ces éléments devraient faire l'objet d'une attention particulière lors de la mise en œuvre de programmes de gestion des maladies chroniques. À cet égard, une plus grande proximité entre équipes interdisciplinaires et médecins de première ligne, ce que préconisent les nouveaux modèles d'organisation de première ligne où ces équipes existent, constitue une voie à privilégier en matière de gestion des maladies chroniques.

\section{Remerciements}

Le projet de recherche dans lequel s'inscrit le contenu de cet article a été financé par les Instituts de recherche en santé du Canada (Programme de Partenariats pour l'amélioration des systèmes de santé) et le Fonds Pfizer-FRSQ (Fonds de recherche en santé du Québec)-MSSS (Ministère de la Santé et des Services sociaux) sur les maladies chroniques. Les auteurs aimeraient souligner la contribution des collaborateurs associés au projet à l'Agence de la santé et des services sociaux de Montréal et dans les Centres de santé et des services sociaux participants (Sud-Ouest-Verdun, Jeanne-Mance, Cœur-de-l'île, Pointe-de-l'île, St-Léonard-StMichel et Bordeaux-Cartierville-St-Laurent). 


\section{Conflits d'intérêts}

Les auteurs déclarent n'avoir aucun conflit d'intérêt en lien avec cet article.

\section{Contributions des auteurs}

Sylvie Provost, Raynald Pineault, Dominique Grimard, Pierre Tousignant, Johanne Desforges et Roxane Borgès Da Silva ont participé à la conception et à la conceptualisation de l'étude, à la collecte, à l'analyse et à l'interprétation des données. José Pérez, Michel Fournier et Yves Lévesque ont apporté une contribution majeure à la réalisation des analyses. Tous les auteurs ont contribué à la rédaction de l'article, ont lu et approuvé le manuscrit final.

\section{Références}

1. Wagner, EH. The role of patient care teams in chronic disease management. BMJ. 2000;320(7234):569-572.

2. Von Korff M, Glasgow RE, Sharpe M. Organising care for chronic illness. BMJ. 2002;325(7355):92-94.

3. Rothman AA, Wagner EH. Chronic illness management: what is the role of primary care? Ann Intern Med. 2003; 138(3):256-261.

4. Wagner EH, Austin BT, Davis C, Hindmarsh M, Schaefer J, Bonomi A. Improving chronic illness care: translating evidence into action. Health Aff. 2001;20(6):64-78.

5. Nutting PA, Dickinson WP, Dickinson LM, et al. Use of Chronic Care Model elements is associated with higherquality care for diabetes. Ann Fam Med. 2007;5(1):14-20.

6. Bodenheimer T, Wagner EH, Grumbach K. Improving primary care for patients with chronic illness: the Chronic Care Model, Part 2. JAMA. 2002;288(15): 1909-1914.

7. Coleman K, Austin BT, Brach C, Wagner EH. Evidence on the Chronic Care Model in the new millennium. Health Aff. 2009;28(1):75-85. doi: 10.1377/hlthaff.28.1.75.

8. Glasgow RE, Orleans CT, Wagner EH, Curry SJ, Solberg LI. Does the Chronic Care Model serve also as a template for improving prevention? Milbank Q. 2001;79(4):579-612.
9. Tsai AC, Morton SC, Mangione CM, Keeler EB. A meta-analysis of interventions to improve care for chronic illnesses. Am J Manag Care. 2005;11(8): 478-88.

10. Breton $\mathrm{M}$, Denis JL, Lamothe L. Incorporating public health more closely into local governance of healthcare delivery: lessons from the Québec experience. Can J Public Health. 2010; $101(4): 314-317$.

11. Breton M, Pineault R, Levesque JF, Roberge $\mathrm{D}$, Borgès Da Silva $\mathrm{R}$, Prud'homme A. Reforming healthcare systems on a locally integrated basis: is there a potential for increasing collaborations in primary healthcare? BMC Health Serv Res. 2013;13(262). doi: 10.1186/1472-6963-13-262.

12. Bodenheimer T, Wagner EH, Grumbach K. Improving primary care for patients with chronic illness. JAMA. 2002; 288(14):1175-1179.

13. Grumbach K. Chronic illness, comorbidities, and the need for medical generalism. Ann Fam Med. 2003;1(1):4-7. doi: $10.1370 / \mathrm{afm} .47$.

14. Starfield B, Lemke KW, Bernhardt T, Foldes SS, Forrest CB, Weiner JP. Comorbidity: implications for the importance of primary care in "case" management. Ann Fam Med. 2003; 1(1):8-14. doi: 10.1370/afm.1.

15. Reeve J, Blakeman T, Freeman GK, et al. Generalist solutions to complex problems: generating practice-based evidence-the example of managing multi-morbidity. BMC Fam Pract. 2013;14(112). doi: 10.1186/1471-2296 $-14-112$

16. Provost $S$, Borgès Da Silva $R$, Pineault R, Grimard D, Pérez J, Tousignant P. Mise en œuvre d'un programme de prévention et d'intervention interdisciplinaire intégré sur le risque cardiométabolique en première ligne : le point de vue des médecins participants. Présentation par affiche. Résumé \#70. Conférence de l'ACRSPS, mai 2016; Toronto, Canada.

17. Provost S, Pineault R, Tousignant P, Hamel $M$, Borgès Da Silva R. Evaluation of the implementation of an integrated primary care network for prevention and management of cardiometabolic risk in Montréal. BMC Fam Pract. 2011;12(126). doi: 10.1186/1471 -2296-12-126.
18. Nolin B, Prud'homme D, Godin G, Hamel D. Enquête québécoise sur l'activité physique et la santé 1998. Québec (Québec) : Institut de la statistique du Québec, Institut national de santé publique du Québec et KinoQuébec; 2002.

19. Ministère de la Santé et des Services sociaux. Coup d'œil sur l'alimentation de la personne diabétique [Internet]. Québec (Québec) : Ministère de la Santé et des Services sociaux; 2014. En ligne à : http://www.diabete.qc.ca/fr /comprendre-le-diabete/ressources /getdocumentutile/coup-oeil-diabeteFR .pdf

20. Bradley C, Todd C, Gorton T, Symonds E, Martin A, Plowright R. The development of an individualized questionnaire measure of perceived impact of diabetes on quality of life: the ADDQoL. Qual Life Res. 1999;8(1-2): 79-91.

21. El Achhab Y, Nejjari C, Chikri M, Lyoussi B. Disease-specific healthrelated quality of life instruments among adults diabetic: a systematic review. Diabetes Res Clin Pract. 2008; 80(2):171-84. doi: 10.1016/j.diabres .2007.12.020.

22. Speight J, Reaney MD, Barnard KD. Not all roads lead to Rome: a review of quality of life measurement in adults with diabetes. Diabet Med. 2009;26(4): 315-327. doi: 10.1111/j.1464-5491.2009 .02682.x.

23. Battersby MW, Ask A, Reece MM, Markwick MJ, Collins JP. The Partners in Health scale: the development and psychometric properties of a generic assessment scale for chronic condition self-management. Aust J Prim Health. 2003;9(4):41-52. doi: 10.1071/PY03022.

24. Stellefson M, Dipnarine K, Stopka C. The Chronic Care Model and diabetes management in US primary care settings: a systematic review. Prev Chronic Dis. 2013;10:120180. doi: 10.5888/pcd10.120180.

25. Elissen AM, Steuten LM, Lemmens LC, Drewes HW, Lemmens KM, Meeuwissen JA, et al. Meta-analysis of the effectiveness of chronic care management for diabetes: investigating heterogeneity in outcomes. J Eval Clin Pract. 2013;19(5),753-762. doi: 10.1111 /j.1365-2753.2012.01817.x. 
26. Andridge RR, Little RJ. A review of hot deck imputation for survey nonresponse. Int Stat Rev. 2010;78(1): 40-64. doi: 10.1111/j.1751-5823.2010 .00103.x.

27. Haziza D. Chapter 10: Imputation and inference in the presence of missing data. Dans : Pfeffermann D, Rao CR (dir.). Handbook of Statistics 29A: Sample surveys: design, methods and applications. Oxford (R.-U.) : Elsevier; 2009:215-246. 